\title{
Factores de riesgo para amputación en pacientes manejados con fasciotomía de miembro inferior en un centro de alta complejidad
}

\author{
David Muñoz P. ${ }^{1}$, Rolando Medina R. ${ }^{1}$ y Juan Sanjuan M. ${ }^{1}$
}

1 Universidad Surcolombiana

(USCO), Hospital Universitario Hernando Moncaleano Perdomo, Neiva. Huila, Colombia.

Recibido 2019-12-26 y aceptado 2020-02-15

Correspondencia a: Dr. David Felipe Muñoz P. pipetrored@gmail.com
Risk factors for amputation in patients managed with lower limb fasciotomy in a high complexity center

Introduction: The lower limb compartment syndrome has the potential to cause devastating morbidity in patients and high legal medical risks for doctors involved in its treatment. Once established, fasciotomy is the only effective treatment. The loss of the affected limb is the complication with a greater burden of disease. There are few descriptions of risk factors for the need for lower limb amputation after having undergone fasciotomy in patients with traumatic injuries. Materials and Method: A retrospective, observational, analytical study was conducted in which information was collected from patients with lower limb trauma that required thigh or leg fasciotomy for a period of 10 years in search of factors that could influence limb loss. Results: 21 patients met the inclusion criteria of which $6(28.57 \%)$ were amputated and 2 died $(9.52 \%)$. The majority of the individuals were under 30 years old and almost all of the male sex. We found that the percentage of amputation seems to be affected statistically significantly by factors such as a high ISS (mean of 24), paresthesia at admission, performing late fasciotomy ( $>6 \mathrm{~h}$ ), muscle reactivity at the time of surgery, postoperative SSI and reintervention by vascular graft thrombosis. Conclusions: We found risk factors that may indicate the loss of the lower limb after being subjected to fasciotomy in the context of trauma. A prospective follow-up and a greater number of patients could make it possible to elucidate more of these factors.

Key words: fasciotomy; amputation; compartment syndrome.

\section{Resumen}

Introducción: El síndrome compartimental del miembro inferior tiene el potencial de causar morbilidad devastadora en los pacientes y altos riesgos médico-legales para los médicos involucrados en su tratamiento. Una vez instaurado, la fasciotomía se constituye como el único tratamiento efectivo. La pérdida de la extremidad afectada es su complicación con mayor carga de enfermedad. Existen pocas descripciones sobre factores de riesgo para la necesidad de amputación de miembro inferior luego de haber sido sometido a fasciotomía en pacientes con lesiones traumáticas. Materiales y Método: Se realizó un estudio retrospectivo, observacional, analítico en el cual se recolectó información de pacientes con traumatismo de miembro inferior que requirieron fasciotomía de muslo o pierna durante un periodo de 10 años en busca de factores que pudieron influir en la pérdida de la extremidad. Resultados: 21 pacientes cumplían los criterios de inclusión de los cuales $6(28,57 \%)$ fueron amputados y 2 fallecieron (9,52\%). La mayoría de los individuos fueron menores de 30 años y casi la totalidad del sexo masculino. Encontramos que el porcentaje de amputación parece verse afectado de manera estadísticamente significativa por factores como un International Severity Score (ISS) elevado (media de 24), las parestesias al ingreso, la realización de fasciotomía tardía (>6 h), la reactividad muscular al momento de la cirugía, la infección del sitio operatorio y la reintervención por trombosis del injerto vascular. Conclusiones: Existen factores de riesgo que pueden indicar la pérdida de la extremidad inferior luego de ser sometido a fasciotomía en el contexto de trauma. Un seguimiento prospectivo y un mayor número de pacientes podrían permitir dilucidar más de dichos factores.

Palabras clave: fasciotomía; amputación, síndrome compartimental. 


\section{Introducción}

El síndrome compartimental del miembro inferior tiene el potencial de causar morbilidad devastadora en los pacientes y altos riesgos médico-legales para los médicos involucrados en el tratamiento ${ }^{1-3}$. La incidencia en el mundo occidental es de 3,1 por 100.000 habitantes, los hombres se ven 10 veces más afectados que las mujeres y la edad promedio es de 32 años $^{4-6}$. La fasciotomía es un procedimiento quirúrgico que se realiza una vez se ha instaurado el síndrome compartimental ${ }^{7}$. El procedimiento consiste de una o más incisiones en las fascias que permiten la descompresión de los compartimientos musculares y constituye el único tratamiento efecti$\mathrm{vo}^{8,9}$. La incidencia del uso de fasciotomía varía de acuerdo al mecanismo de trauma siendo de $0,9 \%$ en relación con los accidentes de tránsito y hasta $8,9 \%$ en relación a las heridas por proyectil de arma de fuego $^{3}$. A pesar de la baja incidencia, es fundamental el reconocimiento temprano para la prevención de sus complicaciones ${ }^{10-13}$.

La importancia de una fasciotomía oportuna está orientada a la prevención del síndrome compartimental, siendo el trauma la principal causa de este ${ }^{1,3,8,14}$. La complicación con mayor carga de enfermedad de esta entidad es la pérdida de la extremidad. Las amputaciones se asocian al diagnóstico tardío, el tratamiento inadecuado y la etiología del síndrome compartimental y tienen importantes implicaciones médicas, funcionales, legales y psicológicas $^{2,5,15,18}$. La mortalidad luego de la amputación de miembro inferior es notoriamente alta, siendo del $22 \%-52 \%$ para el primer año, $33 \%$ a 3 años y $39 \%$ a $68 \%$ a 5 años ${ }^{15,19}$. Este fenómeno también ocasiona un alto impacto económico a los sistemas de salud ${ }^{20,21}$.

Previamente han sido descritos factores de riesgo asociados al desarrollo del síndrome compartimental, la necesidad de fasciotomía y la amputación de la extremidad en el contexto del trauma ${ }^{2-4,22-24}$. Sin embargo, existen pocas descripciones sobre factores de riesgo que predigan la necesidad de amputación en pacientes con trauma de miembro inferior sometidos a fasciotomía, en relación a lo cual se planteó el presente estudio.

\section{Materiales y Método}

Se realizó un estudio retrospectivo, observacional, analítico y descriptivo de corte transversal en el periodo comprendido desde el $1^{\circ}$ de enero de 2009 al 31 de enero de 2019, la información se obtuvo del registro de historias clínicas diligenciándose un instrumento creado por los autores. Este estudio se realizó en el Hospital Universitario Hernando Moncaleano Perdomo de Neiva, Colombia, institución de alta complejidad y centro de referencia para buena parte del sur del país. Se incluyeron pacientes mayores de 13 años con traumatismo de miembro inferior a quienes se les realizó fasciotomía de muslo o pierna, fueron excluidos aquellos en los cuales no se encontró información relacionada a la evolución clínica de la extremidad. Este estudio fue aprobado por el comité de ética institucional.

Los datos fueron capturados mediante Google Form, luego se realizó un análisis exploratorio Excel $2013^{\circledR}$ y posteriormente importados a Stata $15^{\circledR}$. Se consideró como significancia estadística un valor de $\mathrm{p}<0,05$.

\section{Resultados}

Durante el periodo de 10 años (2009-2019) considerado para el estudio se encontraron 40 pacientes llevados a fasciotomía de muslo o pierna en nuestra institución. Los criterios de inclusión establecidos se cumplieron en 21 pacientes. Ningún paciente presentó dos o más fasciotomías concomitantes en ambos miembros, por lo tanto, en este artículo todas las estadísticas se referirán a pacientes y no a número de miembros inferiores. Las Tablas 1 y 2 resumen características al ingreso de los pacientes incluidos para el análisis.

El 90,48\% de los pacientes fueron de sexo masculino $(\mathrm{n}=19)$. Solo dos $(9,52 \%)$ pacientes del total de la población fallecieron y de estos ninguno fue llevado a amputación. Seis $(28,6 \%)$ pacientes fueron llevados a amputación. Factores como la edad, el tipo de aseguramiento en salud, los antecedentes médicos y el hecho de que el paciente ingresase remitido de otra institución no marcaron diferencias estadísticamente significativas (p: NS).

En el grupo de pacientes llevados a amputación la mayoría sufrió heridas penetrantes (heridas por proyectil de arma de fuego o HPAF, heridas por arma corto punzante o HPAC) y las variables vitales al ingreso se encontraban con mayor frecuencia por fuera de rangos de normalidad (p: NS).

Se midieron también puntajes pronósticos y de severidad comúnmente utilizados en trauma de extremidad. El puntaje International Severity Score (ISS) fue mayor en los pacientes llevados a amputación y este hallazgo fue estadísticamente significativo (mediana ISS $25 \mathrm{RIC}=24-27$ amputa- 
Tabla l. Características de los pacientes al ingreso

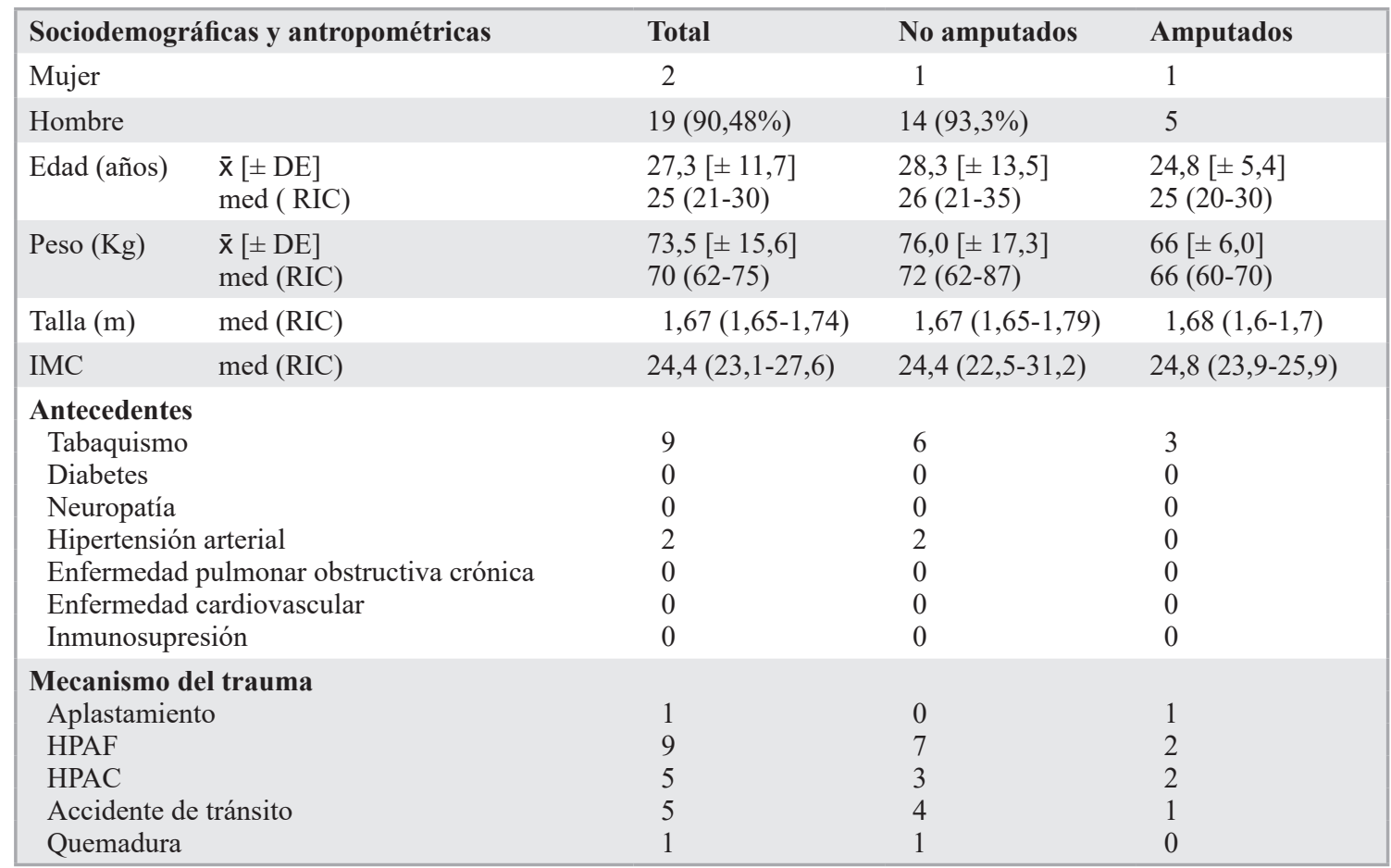

$\overline{\mathrm{X}}$ : media; \pm DE: desviación estandar; med: mediana; RIC: rango intercuartílico; IMC: índice de masa corporal; HPAF: herida por proyectil de arma de fuego; HPAC: herida por arma cortopunzante.

Tabla 2. Signos vitales y otras variables al ingreso

\begin{tabular}{|c|c|c|c|c|c|}
\hline & & & & & Valor $\mathbf{p}$ \\
\hline \multicolumn{6}{|c|}{ Signos vitales (al ingreso) } \\
\hline TAS (mmHg) & $\begin{array}{l}\bar{x}[ \pm \mathrm{DE}] \\
\text { med }(\mathrm{RIC})\end{array}$ & $\begin{array}{l}101[ \pm 36] \\
105(90-128)\end{array}$ & $\begin{array}{l}103,7[ \pm 37,2] \\
105(90-138)\end{array}$ & $\begin{array}{c}94,2[ \pm 35,4] \\
100(60-128)\end{array}$ & \\
\hline TAM (mmHg) & $\begin{array}{l}\bar{x}[ \pm \mathrm{DE}] \\
\text { med }(\mathrm{RIC})\end{array}$ & $\begin{array}{l}75,1[ \pm 25,9] \\
76(78-93)\end{array}$ & $\begin{array}{l}77,6[ \pm 27,8] \\
80(71-103)\end{array}$ & $\begin{array}{l}68,8[ \pm 21,1] \\
74(53-83)\end{array}$ & \\
\hline Fr (rpm) & $\begin{array}{l}\bar{x}[ \pm \mathrm{DE}] \\
\text { med }(\mathrm{RIC})\end{array}$ & $\begin{array}{l}22,57[ \pm 5,7] \\
20(19-26)\end{array}$ & $\begin{array}{l}21,73[ \pm 5,2] \\
20(18-26)\end{array}$ & $\begin{array}{l}24,66[ \pm 6,9] \\
25(19-32)\end{array}$ & \\
\hline $\mathrm{Fc}(\mathrm{lpm})$ & $\begin{array}{l}\bar{x}[ \pm \mathrm{DE}] \\
\text { med }(\mathrm{RIC})\end{array}$ & $\begin{array}{l}103,9[ \pm 19,6] \\
100(90-115)\end{array}$ & $\begin{array}{c}101,6[ \pm 20,5] \\
97(90-105)\end{array}$ & $\begin{array}{l}109,6[ \pm 17,4] \\
108(100-125)\end{array}$ & \\
\hline \multicolumn{6}{|l|}{ Otras variables } \\
\hline Remitido de ot & titución & $15(71,43 \%)$ & $11(73,3 \%)$ & 4 & \multirow{4}{*}{0,021} \\
\hline Glasgow & med (RIC) & $15(14-15)$ & $15(14-15)$ & $15(13-15)$ & \\
\hline ISS & $\begin{array}{l}\bar{x}[ \pm \mathrm{DE}] \\
\text { med (RIC) }\end{array}$ & $\begin{array}{l}18,6[ \pm 6,4] \\
16(16-25)\end{array}$ & $\begin{array}{l}16,5[ \pm 5,9] \\
16(13-18)\end{array}$ & $\begin{array}{l}24[ \pm 4] \\
25(24-27)\end{array}$ & \\
\hline MESS & $\begin{array}{l}\bar{x}[ \pm \mathrm{DE}] \\
\text { med (RIC) }\end{array}$ & $\begin{array}{l}5,3[ \pm 2,3] \\
7(7-7)\end{array}$ & $\begin{array}{l}4,5[ \pm 2] \\
7(7-7)\end{array}$ & $\begin{array}{l}7,5[ \pm 1] \\
7(5,8-7)\end{array}$ & \\
\hline \multicolumn{6}{|c|}{ AIS extremidades } \\
\hline 2 & & 1 & 1 & 0 & \\
\hline 3 & & 5 & 5 & 0 & \\
\hline 4 & & $12(57,14 \%)$ & 8 & 4 & \\
\hline 5 & & 3 & 1 & 2 & \\
\hline
\end{tabular}

X̄: media; \pm DE: desviación estandar; med: mediana; RIC: rango intercuartílico; TAS: tensión arterial sistólica; TAM: tensión arterial media; Fr: frecuencia respiratoria; Fc: frecuencia cardiaca; ISS: international severity score; MESS: mangled extremity severity score; AIS: abreviated injury score. 
dos $v s$ mediana ISS 16 RIC $=13-18$ no amputados $\mathrm{p}=0,02)$. El Mangled Extremity Severity Score (MESS) también fue mayor en los pacientes con pérdida de la extremidad afectada. Además, todos los pacientes amputados presentaron un Abreviated Injury Score (AIS) para las extremidades de 4 o 5 (66,7\% y $33,3 \%$ respectivamente). Sin embargo, las asociaciones no fueron estadísticamente significativas para estos últimos.
Los signos y síntomas asociados con el síndrome compartimental presentes en los pacientes al momento del ingreso se resumen en la Tabla 3. De manera llamativa la parestesia fue un síntoma presente en el 83,3\% $(n=5)$ de los amputados, con una $\mathrm{p}$ válida $(\mathrm{p}=0,029)$.

Las características asociadas a la realización de la fasciotomía y la amputación se encuentran consignadas en la Tabla 4. A ningún paciente se le

Tabla 3. Signos y síntomas de isquemia tisular al ingreso

\begin{tabular}{|lcccc|}
\hline & Total & No amputados & Amputados & Valor p \\
Signos y síntomas de isquemia & & & & \\
Cambios isquémicos cutáneos & $14(66,67 \%)$ & 8 & 6 & \\
Dolor desproporcionado & $10(47,62 \%)$ & 6 & 4 & 0,029 \\
Edema de compartimientos & $16(76,19 \%)$ & $12(80 \%)$ & 5 & 3 \\
Parestesias & 9 & 4 & 3 & \\
Paresias & 7 & 4 & 8 & \\
Ausencia de pulso & $14(66,67 \%)$ & & & \\
\hline
\end{tabular}

Tabla 4. Características de fasciotomía y amputación

\begin{tabular}{|c|c|c|c|c|c|}
\hline & & Total & No amputados & Amputados & Valor $\mathbf{p}$ \\
\hline $\begin{array}{l}\text { Fasciotomía } \\
\text { Profiláctica } \\
\text { Tardía }(>6 \mathrm{~h}) \\
\text { Temprana }(<6 \mathrm{~h})\end{array}$ & & $\begin{array}{c}3 \\
11(52,38 \%) \\
7\end{array}$ & $\begin{array}{l}3 \\
5 \\
7\end{array}$ & $\begin{array}{l}0 \\
6 \\
0\end{array}$ & 0,031 \\
\hline Tiempo a fasciotomía (h) & $\begin{array}{l}\bar{x}[ \pm \text { DE }] \\
\text { med (RIC) }\end{array}$ & $\begin{array}{c}10,61[ \pm 12,8] \\
4(3-12)\end{array}$ & $\begin{array}{c}9,33[ \pm 14,3] \\
3(2-4)\end{array}$ & $\begin{array}{c}13,8[ \pm 7,8] \\
11(9-19)\end{array}$ & 0,03 \\
\hline Diagnóstico Sx compartimen & & $16(76,2 \%)$ & $12(80 \%)$ & 4 & \\
\hline $\begin{array}{l}\text { Nivel de fasciotomía } \\
\text { Muslo } \\
\text { Pierna } \\
\text { Pierna y muslo }\end{array}$ & & $\begin{array}{c}3 \\
16(76,2 \%) \\
2\end{array}$ & $\begin{array}{c}3 \\
12(80 \%) \\
0\end{array}$ & $\begin{array}{l}0 \\
4 \\
2\end{array}$ & \\
\hline $\begin{array}{l}\text { Vibilidad } \\
\text { Hiporreactivo } \\
\text { No reactivo }\end{array}$ & & $\begin{array}{l}5 \\
3\end{array}$ & $\begin{array}{l}1 \\
1\end{array}$ & $\begin{array}{l}4 \\
2\end{array}$ & \\
\hline Reactivo & & $13(61,9 \%)$ & $13(86,7 \%)$ & & 0,001 \\
\hline Amputación & & 6 & $15(100 \%)$ & 6 & \\
\hline Tiempo a amputación (días) & $\begin{array}{l}\bar{x}[ \pm \mathrm{DE}] \\
\operatorname{med}(\mathrm{RIC})\end{array}$ & $\begin{array}{c}7,2[ \pm 4,1] \\
8(3-11)\end{array}$ & & & \\
\hline $\begin{array}{l}\text { Nivel } \\
\text { Desarticulación } \\
\text { Infrapatelar } \\
\text { Suprapatelar } \\
\text { Muerte }\end{array}$ & & $\begin{array}{l}1 \\
2 \\
3 \\
2\end{array}$ & 2 & 0 & \\
\hline Estancia (días) & $\begin{array}{l}\bar{x}[ \pm \text { DE }] \\
\text { med (RIC) }\end{array}$ & $\begin{array}{c}19,8[ \pm 14,3] \\
16(11-21)\end{array}$ & $\begin{array}{c}16,3[ \pm 10,3] \\
14(11-21)\end{array}$ & $\begin{array}{c}28,3[ \pm 20,0] \\
22(16-33)\end{array}$ & \\
\hline
\end{tabular}

$\overline{\mathrm{X}}$ : media; \pm DE: desviación estándar; med: mediana; RIC: rango intercuartílico. 
Tabla 5. Lesiones y complicaciones asociadas

\begin{tabular}{|c|c|c|c|c|}
\hline Lesiones asociadas & Total & No amputados & Amputados & Valor $\mathbf{p}$ \\
\hline $\begin{array}{l}\text { Lesión arterial } \\
\text { Localización }\end{array}$ & $10(47,6 \%)$ & 6 & 4 & \\
\hline Femoral común & 2 & 1 & 1 & \\
\hline Femoral superficial & 3 & 2 & 1 & \\
\hline Poplítea & 2 & 0 & 2 & \\
\hline Tibial posterior & 3 & 3 & 0 & \\
\hline $\begin{array}{l}\text { Lesión venosa } \\
\text { Localización }\end{array}$ & 5 & 3 & 2 & \\
\hline Femoral & 1 & 0 & 1 & \\
\hline Femoral común & 1 & 1 & 0 & \\
\hline Safena mayor & 1 & 0 & 1 & \\
\hline Tibial posterior & 2 & 2 & 0 & \\
\hline Fracturas & 5 & 4 & 1 & \\
\hline Localización & & & & \\
\hline Fémur & 2 & 2 & 0 & \\
\hline Tibia & 3 & 2 & 1 & \\
\hline Clasificación Gustillo & & & & \\
\hline Cerrada & 2 & 2 & 0 & \\
\hline Tipo II & 2 & 2 & 0 & \\
\hline Tipo IIIc & 2 & 1 & 1 & \\
\hline Avulsión de tejidos & 4 & 1 & 3 & \\
\hline ISO & 5 & 1 & 4 & \\
\hline Profunda, cantidad (\%) & 4 & 0 & 4 & 0,003 \\
\hline Superficial, cantidad (\%) & 1 & 1 & 0 & 0,003 \\
\hline
\end{tabular}

ISO: infección del sitio operatorio. realizó diagnóstico mediante medición objetiva de la presión de los compartimientos. La fasciotomía se realizó de manera profiláctica (antes del diagnóstico) o temprana ( $<6 \mathrm{~h}$ del trauma) en el $66,7 \%$ de los pacientes $(\mathrm{n}=10)$ y de estos ninguno fue llevado a amputación. A la totalidad de los pacientes amputados se les realizó fasciotomía terapéutica tardía $(>6 \mathrm{~h})(\mathrm{p}=0,03)$, con un tiempo promedio de $13,83 \mathrm{~h}(\mathrm{p}=0,03)$.

También se evaluó la reactividad del músculo en el momento de la cirugía mediante estímulo eléctrico. Ninguno de los pacientes con adecuada reactividad muscular fue llevado a amputación $(p=0,001)$. En cambio fueron los pacientes con músculos hiperactivos o no reactivos los que mayormente sufrieron pérdida de la extremidad.

Las lesiones asociadas y complicaciones asociadas, al igual que los manejos de las mismas se agruparon en las Tablas 5 y 6 . Todos los pacientes reintervenidos por trombosis de injerto vascular fueron amputados $(\mathrm{p}=>0,001)$.

La presencia de avulsión de tejidos blandos asociada al trauma se registró en el $50 \%(n=3)$ de aquellos que sufrieron pérdida de la extremidad $(\mathrm{p}=\mathrm{NS})$. Otras lesiones asociadas no fueron relevantes en el análisis de los datos.

Se evaluaron posibles complicaciones durante

Tabla 6. Manejo de lesiones y complicaciones asociadas

\begin{tabular}{|c|c|c|c|c|}
\hline & Total & No amputados & Amputados & Valor $\mathbf{p}$ \\
\hline $\begin{array}{l}\text { Lesiones arteriales } \\
\text { Anastomosis } \\
\text { Injerto autólogo } \\
\text { Injerto sintético } \\
\text { Rafia }\end{array}$ & $\begin{array}{l}4 \\
4 \\
1 \\
1\end{array}$ & $\begin{array}{l}3 \\
2 \\
0 \\
1\end{array}$ & $\begin{array}{l}1 \\
2 \\
1 \\
0\end{array}$ & \\
\hline $\begin{array}{l}\text { Lesiones venosas } \\
\text { Anastomosis } \\
\text { Ligadura } \\
\text { Rafia }\end{array}$ & $\begin{array}{l}3 \\
1 \\
1\end{array}$ & $\begin{array}{l}2 \\
0 \\
1\end{array}$ & 1 & \\
\hline $\begin{array}{l}\text { Fracturas } \\
\text { Osteosíntesis } \\
\text { Tutor externo, cantidad (\%) }\end{array}$ & $\begin{array}{l}2 \\
4\end{array}$ & $\begin{array}{l}2 \\
3\end{array}$ & & \\
\hline $\begin{array}{l}\text { Reintervención } \\
\text { Trombosis de anastomosis }\end{array}$ & 1 & 1 & & \\
\hline Trombosis de injerto & 4 & 0 & 4 & $<0,001$ \\
\hline $\begin{array}{l}\text { Otros } \\
\text { GRE } \\
\text { GRE (unidades) } \\
\begin{array}{l}\overline{\mathrm{x}}[ \pm \mathrm{DE}] \\
\operatorname{med}(\mathrm{RIC})\end{array}\end{array}$ & $\begin{array}{l}10(47,6 \%) \\
3,44[ \pm 1,0] \\
\quad 4(3-4)\end{array}$ & $\begin{array}{c}5 \\
3,25[ \pm 0,9] \\
4(3-4)\end{array}$ & $\begin{array}{c}5 \\
3,6[ \pm 1,1] \\
4(3-4)\end{array}$ & \\
\hline $\begin{array}{l}\text { Vasopresor, cantidad (\%) } \\
\text { UCI, cantidad (\%) }\end{array}$ & $\begin{array}{l}6 \\
5\end{array}$ & $\begin{array}{l}3 \\
3\end{array}$ & $\begin{array}{l}3 \\
2\end{array}$ & \\
\hline
\end{tabular}

X̄: media; \pm DE: desviación estándar; med: mediana; RIC: rango intercuartílico. GRE: glóbulos rojos empaquetados. 
la estancia hospitalaria. Todos los pacientes que presentaron infección del sitio operatorio (ISO) profunda, fueron amputados $(\mathrm{p}=0,001)$. La estancia hospitalaria fue mayor para los pacientes que perdieron la extremidad.

Por último se planteó, además, la medición de otros factores que podían influenciar la evolución clínica. En la mitad de los pacientes que recibieron trasfusión de glóbulos rojos empaquetados (GRE) durante las primeras $24 \mathrm{~h}$ hubo fracaso del manejo conservador. Se midió, además, la necesidad de vasopresores y estancia en UCI. Ninguna de estas variables permitió establecer asociaciones estadísticamente significativas.

\section{Discusión}

Nuestro estudio tenía como objetivo principal dilucidar los factores de riesgo para amputación en los pacientes con trauma de extremidad inferior llevados a fasciotomía. Múltiples publicaciones previas apuntaban a la identificación de factores predictores de necesidad de fasciotomía temprana y desenlaces adversos posterior a la realización de esta, sin buscar específicamente factores relacionados a la pérdida de la extremidad.

Bernardino et al. ${ }^{3}$, llevaron a cabo el mayor estudio hasta la fecha para incidencia y caracterización de pacientes llevados a fasciotomía de miembro inferior, identificando la presencia de lesión vascular, requerimiento de trasfusión de glóbulos rojos, sexo masculino, fractura abierta, herida por proyectil de arma de fuego, ISS $>16$ y edad $<55$ años como predictores independientes de necesidad de fasciotomía. Velmahos et al. ${ }^{25}$, estudiaron las complicaciones y los factores de riesgo para presentarlas en fasciotomías asociadas a trauma y reportaron que el riesgo fue mayor en pacientes sometidos a fasciotomía de miembro inferior, procedimiento realizado $>8 \mathrm{~h}$ posterior al trauma y heridas vasculares y musculoesqueléticas asociadas.

Los factores de riesgo que sugieren estas y otras publicaciones pueden agruparse de manera general en aquellos propios del paciente, aquellos relacionados a la mecánica del trauma (incluyendo las lesiones asociadas) y aquellos relacionados a la realización de fasciotomía de miembro inferior propiamente dichos.

En cuanto a los factores propios del paciente, al igual que en el estudio de Bernardino, el mayor porcentaje de nuestros pacientes se encontraba constituido por individuos jóvenes de sexo masculino, en los que comúnmente se describe mayor densidad muscular y menor capacidad de distensión de los compartimientos y, por lo tanto, son más susceptibles a presentar síndrome compartimental ${ }^{26}$. Diferentes antecedentes clínicos incrementan el riesgo de amputación ${ }^{18,19,27,28}$. El tabaquismo fue el único relevante de nuestro análisis, el cual estaba presente en la mitad de los pacientes amputados $(\mathrm{p}=\mathrm{NS})$.

Por otro lado, se han establecido previamente condiciones del paciente al ingreso que permiten predecir necesidad de fasciotomía, amputación y desenlaces adversos. De manera local, Jiménez et al. ${ }^{29}$, presentaron una serie de pacientes con trauma vascular periférico identificando factores de riesgo para pérdida de extremidad en este tipo de pacientes los cuales incluyeron un MESS $>7$ y un ISS $>15$. En concordancia, nuestro análisis encontró que los pacientes amputados presentaron mayor alteración de las variables vitales al ingreso y puntajes más altos en scores de predicción y severidad como el MESS, el AIS y el ISS (mediana 25 RIC $=24-27$ para amputados), este último con un valor $\mathrm{p}$ válido. El hallazgo de que el 66,7\% $(n=4)$ de los pacientes amputados ingresaron remitido de otras instituciones, puede haber contribuido a mayores tiempos de isquemia tisular.

En lo que concierne a los factores de riesgo relacionados al mecanismo del trauma, se ha descrito que aquellos por explosión y aplastamiento tienen el peor pronóstico ${ }^{29,30}$. Solo un individuo en nuestro estudio presentó lesiones por aplastamiento y tuvo fallo del manejo conservador de la extremidad. Los mecanismos relacionados con situaciones de violencia interpersonal fueron más comunes como la herida por proyectil de arma de fuego (HPAF) y la herida por arma corto-punzante (HPAC) presentaron el mayor porcentaje de amputaciones. En ninguno se establecieron valores $\mathrm{p}$ significativos.

En cuanto a lesiones asociadas las fracturas abiertas tipo Gustilo IIIB y IIIC muestran peor desenlace y las heridas severas de tejidos blandos con avulsión y pérdida del tejido constituyen la variable más importante a la hora de influenciar la decisión de amputar e incrementan significativamente el riesgo de complicaciones (infección crónica, dolor, falla de anastomosis) ${ }^{22,31}$. La lesión vascular con isquemia mayor a seis horas aumenta el riesgo de desenlaces adversos si se intenta salvar la extremidad ${ }^{22,30,31}$. En la población de nuestro estudio, se evidenció que los pacientes con trauma vascular arterial aislado presentaron con mayor frecuencia fallo del manejo conservador, sin que la localización anatómica mostrara diferencias. Las fracturas abiertas ni su localización fueron prepon- 
derantes en los pacientes llevados a amputación, pero sí se encontró que los pacientes con avulsión importante de tejidos blandos perdieron la extremidad con mayor frecuencia $(p=N S)$. También encontramos signos clínicos de isquemia tisular prolongada en el $100 \%$ de los pacientes amputados al ingreso y se logró establecer una asociación estadísticamente significativa entre las parestesias y la amputación.

Lazar et al. ${ }^{30}$, analizaron factores de riesgo para amputación en pacientes con lesión arterial describiendo la revascularización fallida, la reintervención y la cirugía tardía. En nuestro análisis de manera significativa se evidenció que el total de los pacientes en quienes se manejó la lesión arterial con injerto (autólogo o sintético) y presentaron reintervención por trombosis del mismo, finalmente fueron amputados, con valor $\mathrm{p}$ válido.

Por último, existen factores asociados a la realización de la fasciotomía que juegan un papel importante en cuanto a la pérdida del miembro comprometido. El retraso en la realización del procedimiento parece ser determinante. Farber et al. ${ }^{32}$, describieron que la fasciotomía temprana $(<6 \mathrm{~h}$ o al momento del reparo vascular) tiene un riesgo 4 veces menor de terminar en amputación, siendo un factor independiente para conservar la extremidad. Otras series de fasciotomías en heridos en combate encontraron resultados similares. En estas, los pacientes que fueron sometidos a fasciotomía temprana tuvieron 50\% menos tasa de amputación que aquellos llevados a fasciotomía tardía ${ }^{33}$. También se ha demostrado que las fasciotomías profilácticas y las realizadas al momento del reparo vascular tienen menor probabilidad de terminar en síndrome compartimental del miembro inferior, que es un factor de riesgo conocido para amputación ${ }^{13,34}$. En la población de nuestro análisis el $80 \%$ de los individuos amputados tenían diagnóstico de síndrome compartimental previo a la cirugía. Ningún paciente sometido a fasciotomía profiláctica perdió la extremidad afectada $\mathrm{y}$, por el contrario, los pacientes amputados fueron en su totalidad llevados a fasciotomía terapéutica posterior a las $6 \mathrm{~h}$ del diagnóstico de síndrome compartimental y/o tuvieron un tiempo a realización de ésta mayor a $11 \mathrm{~h}$. Estos últimos dos valores con valores p estadísticamente significativos. La hiporreactividad y arreactividad muscular fueron hallazgos quirúrgicos también presentes en dicho grupo. La buena reactividad muscular al momento de la cirugía tuvo una asociación estadísticamente significativa con no ser amputado.

Todos los pacientes que en el posoperatorio presentaron ISO perdieron la extremidad, con un valor p estadísticamente válido. La ISO profunda puede haberse precipitado en estos individuos en relación a los hallazgos de tejido desvitalizado durante la realización de la fasciotomía.

La mortalidad global de los pacientes sometidos a fasciotomía se ubica alrededor del 11\%-15\%, la amputación posterior a la fasciotomía se encuentra entre $11 \%-21 \%^{1,3}$. El porcentaje de amputación en nuestro estudio fue ligeramente mayor $(28,57 \%) \mathrm{y}$ la mortalidad fue similar $(9,52 \%)$.

Para nuestro entendimiento, este estudio es el primero que intenta encontrar factores de riesgo de amputación en pacientes llevados a fasciotomía que se realiza a nivel latinoamericano. Sin embargo, por ser de carácter retrospectivo cuenta con limitaciones que podrían ser superados de plantearse un seguimiento prospectivo. El diagnóstico de síndrome compartimental fue realizado en todos los pacientes basados en hallazgos al examen físico, así que no se cuenta con una medición objetiva de la presión intracompartimental. El bajo número de pacientes no permite realizar asociaciones con mayor poder estadístico, pero sienta las bases para análisis posteriores que permitan validar los hallazgos y posibles relaciones que se describen.

\section{Conclusiones}

Nuestro estudio presenta una descripción de las características de los pacientes llevados a amputación luego de ser sometidos a fasciotomía por trauma. Identificamos 21 pacientes llevados a fasciotomía en este contexto, de los cuales $6(28,57 \%)$ fueron amputados y 2 fallecieron $(9,52 \%)$. La mayoría de los individuos fueron menores de 30 años y casi la totalidad del sexo masculino. El porcentaje de amputación parece verse afectado de manera estadísticamente significativa por factores como un ISS elevado (media de 24), las parestesias al ingreso, la realización de fasciotomía tardía $(>6 \mathrm{~h})$ o mayor de $11 \mathrm{~h}$, la reactividad muscular al momento de la cirugía, la ISO en el posoperatorio y la reintervención por trombosis del injerto vascular.

Otros factores que no lograron valores $\mathrm{p}$ significativos pero, presentaron mayor frecuencia de amputación, fueron la mayor alteración de las variables vitales al ingreso, un MESS mayor a 7,5, un AIS extremidad elevado al ingreso, la avulsión importante de tejidos blandos y la trasfusión de hemoderivados en las primeras $24 \mathrm{~h}$. El seguimiento prospectivo podría permitir obtener resultados estadísticamente significativos en este conjunto. 


\section{Responsabilidades éticas}

Protección de personas y animales. Los autores declaran que para esta investigación no se han realizado experimentos en seres humanos ni en animales.
Confidencialidad de los datos. Los autores declaran que en este artículo no aparecen datos de pacientes.

Conflictos de interés: no hay.

\section{Bibliografía}

1. Bowyer MW. Lower Extremity Fasciotomy: Indications and Technique Lower Extremity Fasciotomy: Indications and Technique. Current Trauma Reports 2016;1:35-44. DOI: 10.1007/s40719-0140002-7.

2. Feliciano DV, Cruse PA, Spjut-Patrinely V, Burch JM, Mattox KL. Fasciotomy after trauma to the extremities. Am J Surg. 1988;156:533-6.

3. Branco BC, Inaba $\mathrm{K}$, Barmparas $\mathrm{G}$, Schnüriger $\mathrm{B}$, Lustenberger $\mathrm{T}$, Talving $\mathrm{P}$, et al. Incidence and predictors for the need for fasciotomy after extremity trauma: A 10 -year review in a mature level i trauma centre. Injury 2011;42:1157-63.

4. Duckworth AD, McQueen MM. The Diagnosis of Acute Compartment Syndrome. JBJS Rev. 2017;5:e1.

5. Heemskerk J, Kitslaar P. Acute compartment syndrome of the lower leg: Retrospective study on prevalence, technique, and outcome of fasciotomies. World J Surg. 2003;27:744-7.

6. Cone J, Inaba K. Lower extremity compartment syndrome. Trauma Surg Acute Care Open 2017;2:1-6.

7. Long B, Koyfman A, Gottlieb M. Evaluation and Management of Acute Compartment Syndrome in the Emergency Department. J Emerg Med. 2019;(November 2018):1-12. https://doi. org/10.1016/j.jemermed.2018.12.021.

8. Pechar J, Lyons MM. Acute Compartment Syndrome of the Lower Leg: A Review. J Nurse Pract. 2016;12:265-70.

9. Illical E, Jauregui JJ, Tsai J, Paulino CB, Yarmis SJ, Onuoha KO. Fasciotomy closure techniques. J Orthop Surg. 2017;25:230949901668472.

10. Garner MR, Taylor SA, Gausden E, Lyden JP. Compartment Syndrome: Diagnosis, Management, and Unique Concerns in the Twenty-First Century. HSS J. 2014;10:143-52.

11. González RP, Scott W, Wright A,
Phelan HA, Rodning CB. Anatomic location of penetrating lower-extremity trauma predicts compartment syndrome development. Am J Surg. 2009;197:3715. http://dx.doi.org/10.1016/j. amjsurg.2008.11.013.

12. Kim JYS, Buck DW, Forte AJV, Subramanian VS, Birman MV, Schierle $\mathrm{CF}$, et al. Risk factors for compartment syndrome in traumatic brachial artery injuries: An institutional experience in 139 patients. J Trauma - Inj Infect Crit Care 2009;67:1339-44.

13. Abouezzi Z, Nassoura Z, Ivatury RR, Porter JM, Stahl WM. A critical reappraisal of indications for fasciotomy after extremity vascular trauma. Arch Surg. 1998;133:547-51.

14. Swain R, Ross D. Lower extremity compartment syndrome. Postgrad Med. 2009; 105:159-68.

15. Fortington LV, Geertzen JHB, Van Netten JJ, Postema K, Rommers GM, Dijkstra PU. Short and long term mortality rates after a lower limb amputation. Eur J Vasc Endovasc Surg. 2013;46:124-31. http:// dx.doi.org/10.1016/j.ejvs.2013.03.024

16. Cruz CP, Eidt JF, Capps C, Kirtley L, Moursi MM. Major lower extremity amputations at a Veterans Affairs hospital. Am J Surg. 2003;186:449-54.

17. Schuyler Jones W, Patel MR, Dai D, Vemulapalli S, Subherwal S, Stafford J, et al. High mortality risks after major lower extremity amputation in Medicare patients with peripheral artery disease. Am Heart J. 2013;165:80915.e1. http://dx.doi.org/10.1016/j. ahj.2012.12.002.

18. O'Brien PJ, Cox MW, Shortell CK, Scarborough JE. Risk factors for early failure of surgical amputations: An analysis of 8,878 isolated lower extremity amputation procedures. J Am Coll Surg. 2013;216:836-42.

19. Subramaniam B, Pomposelli F, Talmor D, Park KW. Perioperative and long-term morbidity and mortality after above- knee and below-knee amputations in diabetics and nondiabetics. Anesth Analg. 2005;100:1241-7.

20. Wise ES, McMaster WG, Williamson K, Wergin JE, Hocking KM, Brophy CM. Preoperative Predictors of 30-Day Mortality and Prolonged Length of Stay after Above-Knee Amputation. Ann Vasc Surg. 2016;31:124-33. http://dx.doi. org/10.1016/j.avsg.2015.08.017.

21. Ploeg AJ, Lardenoye JW, Vrancken Peeters MPFM, Breslau PJ.

Contemporary series of morbidity and mortality after lower limb amputation. Eur J Vasc Endovasc Surg. 2005;29:633-7.

22. Scalea TM, DuBose J, Moore EE, West M, Moore FA, McIntyre R, et al. Western Trauma Association Critical Decisions in Trauma: Management of the mangled extremity. J Trauma Acute Care Surg. 2012;72:86-93

23. Johansen K, Daines M, Howey T. Objective criteria accurately predict amputation following lower extremity trauma. J Trauma 1990;30:568-72.

24. Gómez J, Morales C. Fasciotomía profiláctica y síndrome "compartimental" de las extremidades: ¿existen indicaciones justificables? Rev Colomb Cir. 2011;26:101-10. http://ascolcirugia.org/ revista/revistaabriljunio2011/fasciotomia. pdf.

25. Velmahos GC, Theodorou D, Demetriades D, Chan L, Berne TV, Asensio J, et al. Complications and Nonclosure Rates of Fasciotomy for Trauma and Related Risk Factors. World J Surg. 1997;247-53.

26. Long B, Koyfman A, Gottlieb M. Evaluation and Management of Acute Compartment Syndrome in the Emergency Department. J Emerg Med. 2019;56:38697.

27. Rush RM, Arrington ED, Hsu JR. Management of Complex Extremity Injuries. Surg Clin North Am. 2012;92:987-1007. http://dx.doi. org/10.1016/j.suc.2012.06.003. 


\section{ARTÍCULO ORIGINAL}

28. Unwin N. Epidemiology of lower extremity amputation in centres in Europe, North America and East Asia. Br J Surg. 2000;87:328-37. http://www.blackwellsynergy.com/rd.asp?issn=1365-2168\&vol $=87 \&$ page $=328 \&$ goto $=$ abstract.

29. Jiménez HC, Romero E, Medina R, Botache W, Sanjuán J, Martínez C, et al. Caracterización de trauma arterial periférico en un hospital de tercer nivel. Rev Colomb Cirugía 2018;33:2729.

30. Davidovic LB, Cinara IS, Ille T, Kostic
DM, Dragas M V, Markovic DM. Civil and war peripheral arterial trauma: review of risk factors associated with limb loss. Vascular 13:141-7.

31. Rao K, Richardson M, Atkinson N, Brand C, Seneviratine S, Edis D, et al. TRM 07.01 MANGLED LOWER LIMB GUIDELINE Trauma Service Guidelines Title: Mangled Lower Limb Guideline Developed 2014;1-10.

32. Farber A, Tze-Woei T, Hamburg NM. Early Fasciotomy in Patients with Extremity Vascular Injury is Associated with Decreased Risk of Adverse Limb Outcomes: A Review of the National Trauma Data Bank. 2013;43:1486-91.

33. Ritenour AE, Dorlac WC, Fang R, Woods T, Jenkins DH, Flaherty SF, et al. Complications After Fasciotomy Revision and Delayed Compartment Release in Combat Patients. J Trauma Inj Infect Crit Care 2008;64(Supplement):S153-62.

34. Fainzilber G, Roy-Shapira A, Wall MJ, Mattox KL. Predictors of amputation for popliteal artery injuries. Am J Surg. 1995;170:568-71. 